\title{
A study on scrub typhus in children: Experience in a tertiary care hospital
}

\author{
*Soumya Roy ${ }^{1}$, Sumit Datta Majumdar ${ }^{1}$, Subroto Chakrabartty $^{1}$, Swati Chakravarti ${ }^{1}$
}

Sri Lanka Journal of Child Health, 2019; 48(2): 155-159

\begin{abstract}
Objective: To study the epidemiological profile, clinical presentation, laboratory markers, treatment outcomes, complications and prognosis of scrub typhus among children presenting to this tertiary care hospital.
\end{abstract}

Method: A cross sectional study was carried out in a tertiary care paediatric hospital between May 2015 and April 2016 on children between 1 month and 12 years who presented with fever and various clinical features suggestive of rickettsial infections. They were included as a convenience sample and tested for scrub typhus by Weil-Felix test as well as IgM ELISA. Children testing positive for scrub typhus were recruited for further study and observed for the various outcome measures.

Results: There were 136 children between 1 month and 12 years presenting with fever and clinical features suggestive of rickettsial infections during the study period. Of these, 46 children tested positive for scrub typhus and were included in the study. No patient refused to participate or withdrew from the study. The mean age of the children was 5.5 years, $61 \%$ were male, $91 \%$ had rural habitats and admissions peaked between August and December. Mean duration of fever before presentation was 10.3 days. Fever $(100 \%)$, oedema $(45.6 \%)$, irritability (45.6\%), abdominal pain (39.1\%), conjunctival congestion (36.9\%), vomiting (34.8\%), maculopapular rash $(32.6 \%)$, headache $(21.7 \%)$, altered sensorium $(17.4 \%)$, cough (15.2\%), eschar (13\%) and seizure (10.8\%) were the main symptoms. Lymphadenopathy (73.9\%), hepatomegaly $(71.7 \%)$ and splenomegaly $(52.1 \%)$ were the main signs. Elevated C-reactive protein $(100 \%)$, anaemia $(78.3 \%)$, hyponatraemia $(73.9 \%)$, hypoalbuminaemia $(65.2 \%)$, leucocytosis $(56.5 \%)$, transaminitis $(32.6 \%)$ and thrombocytopenia (17.4\%) were important laboratory features. All were IgM ELISA positive

\footnotetext{
${ }^{1}$ Institute of Child Health, Kolkata, India

*Correspondence: dr.roy85@gmail.com

https://orcid.org/0000-0002-4897-6518

(Received on 20 January 2018: Accepted after revision on 24 September 2018)

The authors declare that there are no conflicts of interest Personal funding was used for the project.
}

Open Access Article published under the Creative Commons Attribution CC-BY License and 42 were Weil-Felix test positive. Fever subsided between 24 to 48 hours in the majority. Doxycycline and azithromycin showed similar efficacy. There was no mortality.

Conclusion: Scrub typhus is prevalent among children presenting to this tertiary care hospital in Kolkata, India.

DOI: http://dx.doi.org/10.4038/sljch.v48i2.8712

(Key words: Scrub typhus, children, tertiary hospital)

\section{Introduction}

Scrub typhus has re-emerged to be a global threat in India and globally ${ }^{1}$. Rickettsial infections are often underdiagnosed due to their nonspecific clinical presentation, limited awareness and lack of universal diagnostic facilities. Untreated cases may have fatality rates as high as $30-35 \%{ }^{2}$. We published a small study three years ago, where we tried to demarcate the diverse clinical features of scrub typhus in five children ${ }^{3}$. This study found that except for generalised lymphadenopathy, most features of typhus like continuous fever, apathy, toxaemia and abdominal distension were almost similar to enteric fever, which is also very common in India ${ }^{3}$.

\section{Objective}

To study the epidemiological profile, clinical presentation, laboratory markers, treatment outcomes, complications and prognosis of scrub typhus among the children presenting to this tertiary care hospital.

\section{Method}

A cross sectional observational study was conducted at the Department of Paediatrics, Institute of Child Health, Kolkata, from May 2015 to April 2016. Febrile children, who fulfilled the following inclusion criteria were examined and investigated for rickettsial infection.

Inclusion criteria: Paediatric patients, aged 1 month to 12 years, with fever and one or more of the following: (1) headache / body ache, (2) gastrointestinal symptoms and signs, (3) respiratory symptoms and signs (4) central nervous system symptoms and signs, (5) lymphadenopathy, (6) hepatomegaly, (7) splenomegaly, (8) oedema, (9) rash, (10) eschar, (11) history of tick exposure and (12) recent travel to endemic area. 
Exclusion criteria: Other diagnosed cases of fever not suggestive of rickettsial infections.

Weil-Felix test (WFT) was done in all 136 patients by PROGEN kit manufactured by TULIP DIAGNOSTICS (P) LTD., Verna, Goa, India. A titre of 1:160 or more was taken as positive in each set. Serial dilution of the serum samples in each set was made up as follows: 1:20, 1:40, 1:80, 1:160, $1: 320,1: 640$ and 1:1280. Additionally, IgM ELISA was performed on all of them using scrub Typhus Detect (In Bios International Inc., Seattle, WA, USA) as per the manufacturer's instructions. An optical density (OD) $>0.5$ was considered positive . $^{4}$

Specific antibiotics (doxycycline or azithromycin) were given to the diagnosed rickettsial cases for 7 days. Outcome was evaluated in terms of clinical cure, complications, total hospital stay, need of intensive care and death. The study was approved by the institution's ethics committee and informed consent was obtained from the parents. Statistical analysis was done by using the software statistical version 6 (Tulsa, Oklahoma: Star Soft Inc., 2001).

\section{Results}

A total of 136 febrile children who fulfilled the inclusion criteria were thoroughly examined and investigated for rickettsial infection. Of these 136 children, $46(33.8 \%)$ tested positive for scrub typhus. Of the 46 children, $30(65.2 \%)$ were male and $16(34.8 \%)$ were female. Their mean age was $5.5 \pm 3.4$ years (range 9 to 177 months). Forty two $(91.3 \%)$ were from the rural area. Only $6(13 \%)$ had a definite history of pet exposure (dog in 5 cases and cow in 1 case). There was no history of tick exposure or travel to an endemic zone. These cases were observed between the months of May and December, with a peak from August to December. The majority of children $(91.3 \%)$ had fever for 7-14 days before admission. The average duration of fever was $10.28 \pm 2.5$ days. Important signs and symptoms are described in Table 1 along with a comparison with some other studies on scrub typhus in Indian children ${ }^{5-9}$. Irritability $(45.6 \%)$ and abdominal swelling (32.6\%) were notable features. Laboratory investigations have been described in Tables 1 and 2. Raised C-reactive protein was seen in all cases.

Table 1: Symptoms, signs and laboratory features of the scrub typhus patients $(n=46)$

\begin{tabular}{|c|c|c|c|c|c|c|}
\hline Feature & Our study & Sankhayan et al & Kumar et al & Dass et al & Digra et al & Rajendran et al \\
\hline Male: female & $30: 16$ & $9: 6$ & $20: 15$ & $54: 46$ & $11: 10$ & $12: 6$ \\
\hline Mean duration of fever (days) & 10 & 7 & - & 8.4 & 8 & 10 \\
\hline Mean age (month) & 66 & 78 & 75.6 & 112.8 & - & 104.4 \\
\hline Headache & $10(21.7 \%)$ & - & 11 & 25 & - & 17 \\
\hline Vomiting & $16(34.8 \%)$ & 67 & 49 & 20.8 & - & 44 \\
\hline Altered sensorium & $08(17.4 \%)$ & 73 & 23 & 16.6 & 19 & - \\
\hline Seizure & $05(10.8 \%)$ & 60 & 11 & 12.5 & - & - \\
\hline Cough & $07(15.2 \%)$ & 33 & 51 & 35.7 & - & 28 \\
\hline Conjunctival congestion & $17(36.9 \%)$ & 33 & - & 8.3 & 46 & 34 \\
\hline Abdominal pain & $18(39.1 \%)$ & - & 34 & 25 & 23.8 & 61 \\
\hline Oedema & $21(45.6 \%)$ & 60 & 60 & - & 57 & - \\
\hline Rash & $15(32.6 \%)$ & 67 & 20 & - & 100 & 22 \\
\hline Eschar & $06(13.0 \%)$ & 13 & 11 & 41.7 & - & 72 \\
\hline Lymphadenopathy & $34(73.9 \%)$ & 40 & 37 & 12.5 & 61.9 & 61 \\
\hline Hepatomegaly & $33(71.7 \%)$ & 93 & 91 & 33.3 & 76.2 & 72 \\
\hline Splenomegaly & $24(52.1 \%)$ & 87 & 60 & 45.8 & 76.2 & 88 \\
\hline meningeal irritation & $05(10.9 \%)$ & 33 & 6 & 29.2 & 19 & 0 \\
\hline Anaemia & $36(78.3 \%)$ & - & - & - & 100 & 44 \\
\hline Leucocytosis & $26(56.5 \%)$ & 47 & 37 & 25 & 28.6 & 28 \\
\hline Thrombocytopenia & $08(17.4 \%)$ & 47 & 31 & 26 & 38.1 & 55 \\
\hline Transaminitis & $15(32.6 \%)$ & 100 & 31 & 58.3 & 14.3 & 88 \\
\hline Hyponatraemia & $34(73.9 \%)$ & 53 & 17 & 66.7 & - & - \\
\hline Hypoalbuminaemia & $30(65.2 \%)$ & 100 & 54 & 52.2 & - & 72 \\
\hline
\end{tabular}

Table 2: Laboratory investigation results $(n=46)$

\begin{tabular}{|l|c|}
\hline \multicolumn{1}{|c|}{ Laboratory investigation } & Mean \pm standard deviation (range) \\
\hline Haemoglobin $(\mathrm{g} / \mathrm{dl})$ & $9.83 \pm 1.337(6.40-12.50)$ \\
\hline White blood cell count $($ per cu $\mathrm{mm})$ & $12893.48 \pm 5768.281(4500-28200)$ \\
\hline Platelet count $($ per cu $\mathrm{mm})$ & $161339.13 \pm 78678.780(11000-390000)$ \\
\hline Albumin $(\mathrm{g} / \mathrm{dl})$ & $2.92 \pm 0.449(2.10-4.00)$ \\
\hline Sodium $(\mathrm{mEq} / \mathrm{L})$ & $132.87 \pm 3.436(126-143)$ \\
\hline C-reactive protein $(\mathrm{mg} / \mathrm{L})$ & $76.05 \pm 68.416(5.30-290.20)$ \\
\hline Serum alanine aminotransferase $(\mathrm{IU} / \mathrm{L})$ & $68.22 \pm 45.253(12-231)$ \\
\hline Immunoglobulin M & $2.59 \pm 0.577(0.92-3.73)$ \\
\hline
\end{tabular}


Chest x-ray showed consolidation in 3 cases and pleural effusion in one case. Ultrasonography showed hepato-splenomegaly in 14 cases, isolated splenomegaly in one case and ascites in one case. Cerebrospinal fluid (CSF) examination showed aseptic meningitis in five cases. Echocardiography showed pericardial effusion in one case and myocarditis in another. There was disseminated intravascular coagulation (DIC) in one case, haemophagocytic lymphohistiocytosis (HLH) in two cases and acute disseminated encephalomyelitis (ADEM) like features in another.

WFT and scrub typhus IgM ELISA were done simultaneously on all 136 clinically suspected cases. Only 46 cases tested positive for scrub typhus infection on IgM ELISA test. Mean value of IgM was $2.59 \pm 0.577$ (range $0.92-3.73$ ). In the 46 IgM ELISA positive patients, OX $\mathrm{K}$ was positive $(\geq 1: 160)$ in 42 cases while the remaining 4 cases had OX K titre $<1: 160$. That is, WFT was negative in 4 patients with positive IgM ELISA (Table 3). OX 19 and OX 2 titres were $<1: 160$ in all the patients except one, in whom the OX 2 titre was 1:160. The remaining 90 cases, who were negative for IgM ELISA, were also WFT negative.

Table 3: Titres of OX $K$ in Weil Felix test $(n=46)$

\begin{tabular}{|c|c|}
\hline OX K titre & No. of patients (\%) \\
\hline $1: 640$ & $05(10.9)$ \\
\hline $1: 320$ & $15(32.6)$ \\
\hline $1: 160$ & $22(47.8)$ \\
\hline $1: 80$ & $03(06.5)$ \\
\hline$<1: 20$ & $01(02.2)$ \\
\hline
\end{tabular}

Doxycycline was used in $35(76 \%)$ cases. Out of these, 17 cases received intravenous doxycycline while the other 18 received oral doxycycline. Azithromycin was used in the remaining 11 (24\%) cases. Out of these, only 2 cases received intravenous azithromycin. After the 1st dose of antibiotic, fever subsided within 24 hours in 10 patients $(21.7 \%)$, between 24 to $<48$ hours in 24 $(52.2 \%)$ patients and between 48 to $<72$ hours in 12 patients $(26.1 \%)$. The responses to doxycycline and azithromycin were roughly similar (Table 4).

Table 4: Time taken to become afebrile after first dose of antibiotics

\begin{tabular}{|l|c|c|c|}
\hline $\begin{array}{l}\text { Time taken to become afebrile } \\
\text { after starting antibiotic }\end{array}$ & $\begin{array}{l}\text { Among patients treated } \\
\text { with doxycycline }(\mathbf{n}=\mathbf{3 5})\end{array}$ & $\begin{array}{l}\text { Among patients treated } \\
\text { with azithromycin }(\mathbf{n}=\mathbf{1 1})\end{array}$ & $\begin{array}{l}\text { Total number of } \\
\text { patients }(\mathbf{n}=\mathbf{4 6})\end{array}$ \\
\hline$<24$ hours & $08(22.9 \%)$ & $02(18.2 \%)$ & $10(21.7 \%)$ \\
\hline $24-<48$ hours & $18(51.4 \%)$ & $06(54.5 \%)$ & $24(52.2 \%)$ \\
\hline $48-<72$ hours & $09(25.7 \%)$ & $03(27.3 \%)$ & $12(26.1 \%)$ \\
\hline
\end{tabular}

Fourteen (30.4\%) patients developed complications, aseptic meningitis being the commonest, seen in five $(10.8 \%)$ patients. Pneumonia was seen in three patients while HLH occurred in two patients. Pericardial effusion, myocarditis, pleural effusion, ADEM like features and DIC were seen in one child each. PICU admission was needed for 7 patients and ventilatory support was needed for 2 patients. There was no mortality. The average hospital stay was $7.21 \pm 1.86$ days.

\section{Discussion}

Scrub typhus is prevalent in most parts of the world but is generally difficult to diagnose due to closely similar features with many other tropical infections. It is caused by Orientia tsutsugamushi and is spread by chiggers ${ }^{1}$. In the present study, there were more male than female patients (1.9:1), probably due to the higher prevalence of outdoor sports among boys and consequently, more exposure to chiggers. The majority of cases were between August and December. This is the period when mites are active after the rainy season and is also the breeding season of rodents ${ }^{10}$.

All the children in the present study presented with fever. The duration of fever at the time of presentation was mostly 7-14 days (91.3\%). Besides, pain in the abdomen (39.1\%), vomiting (34.8\%), headache $(21.7 \%)$, body ache (19.5\%), cough $(15.2 \%)$ and convulsions $(10.8 \%)$ were the major associated symptoms. Important signs were generalized lymphadenopathy $\quad(73.9 \%)$, hepatomegaly $(71.7 \%)$, splenomegaly $(52.1 \%)$, oedema $(45.6 \%)$, irritability $(45.6 \%)$ and altered sensorium (17.4\%). Another unique feature observed in this study was the presence of abdominal distension (32.6\%), a feature published by the authors in association with scrub typhus previously too ${ }^{3}$. It is believed to be due to mesenteric artery occlusion (due to vasculitis) giving rise to abdominal angina. Maculopapular rash was found in $15(32.6 \%)$ patients. The painless chigger bite can occur on any part of the body but it is often located in areas that are hard to examine, such as the genital region or under the axilla. An eschar forms at the bite site in about half of the primary infections. It begins as a small papule, enlarges, undergoes central necrosis and acquires a blackened crust. The authors believe that the eschar is due to vasculitis and occlusion of the local skin microvasculature. In our study $6(13 \%)$ patients had eschar, 2 in the genital region, 3 in the trunk and 1 in the face. Eschars have been described as the single most important diagnostic clue ${ }^{10}$. Irritability 
was a very prominent feature (45.6\%), especially among the smaller children. The authors noted that while in enteric fever (the most important differential diagnosis), the child is classically 'toxic and apathetic', in scrub typhus, he is typically 'irritable'. The authors have classically experienced that a child with enteric fever 'hardly cares whatever physical examination is done upon him, unless it is painful' whereas a child with scrub typhus 'actively resents even the approach of the clinician towards him'.

Anaemia, leucocytosis, elevated CRP and liver enzymes, hypoalbuminaemia, hyponatraemia and thrombocytopenia were the prominent features. The complications of scrub typhus noted were aseptic meningitis, pneumonia, pleural effusion, pericardial effusion, myocarditis, HLH and DIC. All these complications have been also reported by Rathi, Dass and others ${ }^{5,11}$. Jang et al. found that the sensitivity and specificity of $\operatorname{IgM}$ ELISA for detection of scrub typhus in comparison with immunofluorescence assay (IFA) (gold standard), were respectively $100 \%$ and $99 \%{ }^{12}$. ELISA does not require any special equipment or sophisticated training and is both rapid and cheap ${ }^{13}$. Rathi et al. suggested that an OD $>0.5$ for IgM by ELISA can be considered positive for scrub typhus ${ }^{11}$.

The poor sensitivity of WFT is well recognised. However Prakash et al. found WFT has a specificity of over $98 \%$ and sensitivity of about $43 \%$ whereas Farhana et al. found them to be $75 \%$ and $79.9 \%$ respectively, when compared with IFA $^{14,15}$. Hence WFT can be used as a reasonably specific screening test, which can be performed rapidly in resource poor settings. WFT has been considered positive with titres of $\geq 1: 160$ based on manufacturers' guidelines. In this present study, 42 out of the 46 patients had OX K titre positive $(\geq$ 1:160). This suggests good correlation between IgM ELISA method and WFT. The American Academy of Paediatrics committee on infectious diseases has identified doxycycline as the drug of choice in rickettsial infection in children of any age $^{16}$. However, Phimda et al. reported doxycycline and azithromycin to be equally efficacious in scrub typhus ${ }^{17}$. Doxycycline was given in a dose of 5 $\mathrm{mg} / \mathrm{kg} /$ day in two divided doses for children below $45 \mathrm{~kg}$ and $200 \mathrm{mg} /$ day in two divided doses for children above $45 \mathrm{~kg}$ for 5-7 days or at least 3 days after defervescence ${ }^{1}$. Due to non-availability of doxycycline or in some cases, when the critical condition of the patient excluded the possibility of waiting for laboratory confirmation, sometimes azithromycin was used based on clinical suspicion $(10 \mathrm{mg} / \mathrm{kg} /$ day once daily). Both doxycycline and azithromycin were found to be almost equally effective. Fever subsided within 48 hours in the majority $(52.2 \%)$ of patients.
This study serves to reflect an experience regarding the clinical features, laboratory markers and treatment as well as prognosis of scrub typhus, which is currently an emerging threat in India and many parts of the world. There were some limitations of the study. It was a hospital-based study and not a community-based one. Serological tests should ideally be done on two serum samples collected at least 14 days apart. Here we have tested only a single serum specimen. IFA or PCR are the gold standard tests whereas we have used WFT and IgM ELISA.

\section{Conclusions}

Scrub typhus is prevalent among children presenting to this tertiary care hospital in Kolkata, India.

\section{References}

1. Rathi N, Rathi A. Rickettsial infections: Indian perspective. Indian Pediatrics 2010; 47(2):157-64. https://doi.org/10.1007/s13312-010-00243

PMid: 20228429

2. Batra, H. Spotted fevers and typhus fever in Tamil Nadu - commentary. Indian Journal of Medical Research 2007; 126: 101-3.

PMid: 17932432

3. Chakrabartty S, Chakravarthy S, Banerjee T, Banerjee C, Bera P, PravinA. Typhus fever - A diagnostic dilemma. Journal of Pediatric Infectious Diseases 2015; 10(01): 32-4.

https://doi.org/10.1055/s-0035-1554973

4. Blacksell SD, Tanganuchitcharnchai A, Nawtaisong P, Kantipong P, Laongnualpanich A, Day NPJ, et al. Diagnostic accuracy of the InBios Scrub Typhus Detect enzyme-linked immunoassay for the detection of $\operatorname{IgM}$ antibodies in northern Thailand. Clinical and Vaccine Immunology 2016; 23:14854.

https://doi.org/10.1128/CVI.00553-15

PMid: 26656118 PMCid: PMC4744921

5. Sankhyan N, Saptharishi LG, Sasidaran K, Kanga A, Singhi SC. Clinical profile of scrub typhus in children and its association with haemophagocytic lymphohistiocytosis. Indian Pediatrics 2014; 51(8):651-3. 
https://doi.org/10.1007/s13312-014-04704

PMid: 25129000

6. Dass R, Deka NM, Duwarah SG, Barman H, Hoque R, Mili D, et al. Characteristics of paediatric scrub typhus during an outbreak in the North Eastern region of India: peculiarities in clinical presentation, laboratory findings and complications. Indian Journal of Pediatrics 2011; 78(11):1365-70.

https://doi.org/10.1007/s12098-011-04705

PMid: 21630069

7. Digra SK, Saini GS, Singh V, Dutt Sharma S, Kaul R. Scrub typhus in children: Jammu Experience. JK Science 2010; 12(2):95-7.

8. Kumar M, Krishnamurthy S, Delhikumar CG, Narayanan P, Biswal N, Srinivasan S. Scrub typhus in children at a tertiary hospital in southern India: clinical profile and complications. Journal of Infection and Public Health 2012; 5(1):82-8. https://doi.org/10.1016/j.jiph.2011.11.001 PMid: 22341847

9. Rajendran A. Scrub typhus in paediatric age group: A report from a tertiary care hospital. Journal of Pediatric Sciences 2011; 3(2):e82.

10. Somu S, Desingh S. The eschar of scrub typhus. Indian Journal of Pediatrics \# (epub ahead of print).

https://doi.org/10.1007/s12098-010-01573

11. Rathi N, Rathi A, Goodman M, Aghai Z. Rickettsial diseases in central India: Proposed clinical scoring system for early detection of spotted fever. Indian Pediatrics 2011; 48(11):867-72. https://doi.org/10.1007/s13312-011-01417

PMid: 21555807

12. Jang W, Huh M, Park K, Choi M, Kim I. Evaluation of an immunoglobulin $\mathrm{M}$ capture enzyme-linked immunosorbent assay for diagnosis of Orienta tsutsugamushi infection. Clinical and Diagnostic Laboratory Immunology 2003; 10:394-8.

PMid: 12738637 PMCid: PMC154952

13. Kalal B, Puranik P, Nagaraj S, Rego S,Shet A. Scrub typhus and spotted fever among hospitalised children in South India: Clinical profile and serological epidemiology. Indian Journal of Medical Microbiology 2016; 34(3):293-8. https://doi.org/10.4103/0255-0857.188315 PMid: 27514949

14. Prakash J, Abraham O, Mathai E. Evaluation of tests for serological diagnosis of scrub typhus. Tropical Doctor 2006; 36:212-14. https://doi.org/10.1258/004947506778604 715

PMid: 17034691

15. Farhana A, Bali N, Kanth F, Farooq R, Haq I, Shah P. Serological evidence of scrub typhus among cases of PUO in the Kashmir Valley- A hospital based study. Journal of Clinical and Diagnostic Research 2016; 10(5):24-6. https://doi.org/10.7860/JCDR/2016/18471 .7825

16. AAP Committee on Infectious Diseases. Rocky Mountain Spotted Fever. In: Red Book. 27th Ed. Elk Grove Village, IL: AAP; 2006. P. 570-2.

17. Phimda K, Hoontrakul S, Suttinont C, Chareonwat S, Losuwanaluk K, Chueasuwanchai $\mathrm{S}$ et al. Doxycycline versus azithromycin for treatment of leptospirosis and scrub typhus. Antimicrobial Agents and Chemotherapy 2007; 51(9):3259-63.

https://doi.org/10.1128/AAC.00508-07

PMid: 17638700 PMCid: PMC2043199 